\title{
Graves' disease associated with juvenile idiopathic arthritis
}

\author{
Vanessa de Matos Santos Mendonça Marques ${ }^{1}$, Sónia CristinaDias de Carvalho², \\ Ana Maria Magalhães Madeira de Paiva Antunes ${ }^{3}$, Olinda Amélia Miranda Cerqueira Castro Pinho Marques, \\ Maria Helena Fernandes Silva ${ }^{5}$, Maria José da Costa Vieira ${ }^{2}$
}

\begin{abstract}
The authors report the case of a 10-year-old girl with Graves' disease (GD), treated with propylthiouracil, who developed uveitis and polyarticular arthritis, and whose mother also had GD and discoid lupus. The differential diagnosis of inflammatory arthritis that appears in a child with autoimmune thyroid disease managed with antithyroid drugs is discussed.
\end{abstract}

Keywords: Graves' disease, arthritis, uveitis, autoimmune diseases, child.

[Rev Bras Reumatol 2011;51(2):184-189] CElsevier Editora Ltda.

\section{INTRODUCTION}

Graves' disease (GD) is an autoimmune disease caused by thyroid stimulating autoantibodies and is characterized by typical symptoms of hyperthyroidism (emotional lability, fatigue, tremor, palpitations) and extrathyroidal manifestations, including ophthalmopathy, myxedema, and acropachy. It can be associated with other autoimmune diseases, particularly systemic lupus erythematous (SLE) and rheumatoid arthritis (RA). ${ }^{1}$ Although rarely, antithyroid drugs, namely propylthiouracil (PTU), are known to be able to induce autoimmune complications like arthritis and vasculitis. ${ }^{2}$

We report the case of a child with GD, who developed juvenile idiopathic arthritis (JIA), and discuss potential causes of arthritis in a child undergoing treatment with PTU.

\section{CASE REPORT}

A 10-year-old girl, first daughter of non-consanguineous parents, presented GD at the age of three and has been treated since then with PTU $(25 \mathrm{mg} /$ day $)$. Her past and family history was unremarkable, except for her mother, who also had GD.

At the age of six, left chorioretinitis of unknown etiology was diagnosed and managed with intravenous corticosteroids.

At the age of seven, the patient was admitted to the Pediatric Department with pain and edema of the left knee for three weeks then, with later involvement of the other knee and tibiotarsal joints, which precluded walking. No fever, no rash, and no respiratory nor gastrointestinal complains were reported.

Received on 1/17/2010. Approved on 1/14/2011. Authors declare no conflict of interest.

Centro Hospitalar do Médio Ave - Unidade de Famalicão - Hospital de S. Marcos - Braga, Portugal.

1. M.D. - Complementary Internship in Pediatrics - Centro Hospitalar do Médio Ave - Famalicão

2. M.D. - Hospital Assistant of Pediatrics and Pediatric Rheumatology - Centro Hospitalar do Médio Ave - Famalicão

3. M.D. - Hospital Assistant of Pediatrics - Hospital de S. Marcos - Braga

4. M.D. - Hospital Assistant of Ophthalmology - Hospital de S. Marcos - Braga

5. M.D. - Hospital Assistant of Endocrinology - Hospital de S. Marcos - Braga

Correspondence to: Sónia Carvalho. Serviço de Pediatria, C.H. Médio-Ave- Unidade de Famalicão - 4761-917 V.N.Famalicão, Portugal. Phone.: 00351 918822430

E-mail: sonia070673@yahoo.com.br 
Clinical examination showed symmetric inflammatory signs in her knees, ankles, metatarsophalangeal joints, and wrists. In addition, pretibial edema, without dermopathy, anterior left uveitis with conjunctival hyperemia, and bilateral exophthalmos with eyelid retraction were also observed.

Laboratory investigation revealed the following: microcytic normochromic anemia (hemoglobin: $9.5 \mathrm{~g} / \mathrm{dL}$ ); thrombocytosis (460,000 platelets/uL); elevated Westergreen erythrocyte sedimentation rate (ESR) $(101 \mathrm{~mm} / \mathrm{h})$; negative antinuclear antibodies (ANA), anti-myeloperoxidase antibodies (MPOANCA), anti-double-stranded DNA antibodies (anti-dsDNA), and rheumatoid factor (RF); elevated gamma globulin; and normal thyroid function.

Clinical improvement of arthritis treated with deflazacort $(1 \mathrm{mg} / \mathrm{kg} / \mathrm{d})$ and ibuprofen $(40 \mathrm{mg} / \mathrm{kg} / \mathrm{d})$ and resolution of uveitis (managed with topical cyclopentolate and corticosteroids) were seen three months later. On this occasion, normalization of the ESR, hemoglobin level, and platelet count was observed; ANCA and anti-dsDNA antibodies remained negative.

The corticosteroids were tapered to one quarter of their initial dose in alternating days, when arthritis relapsed in both knees and tibiotarsal joints. On that occasion, ESR was $84 \mathrm{~mm} / \mathrm{h}$, and, for the first time, MPO-ANCA was positive, but the levels of RF, ANA, and anti-dsDNA antibodies were negative. Therapy with methotrexate was started $\left(15 \mathrm{mg} / \mathrm{m}^{2} /\right.$ week) with marked and sustained clinical improvement.

Currently, at the age of ten, the young girl remains clinically asymptomatic on methotrexate, and, curiously, at this time her mother was diagnosed with discoid lupus.

\section{DISCUSSION}

The appearance of polyarthritis in a child with GD managed with PTU points to some difficulties in the differential diagnosis. These difficulties are due to the fact that arthritis associated with positive MPO-ANCA can be induced by PTU itself. ${ }^{3,4}$ However, the remission of symptoms and the absence of other vasculitic manifestations or ANCA positivity without stopping PTU do not support that hypothesis. Arthritis associated with inflammatory bowel disease is another possibility, but there were neither gastrointestinal nor mucocutaneous manifestations in four years of followup, making this theory less probable. Another pertinent and controversial aspect is the fact that hyperthyroidism, by itself, can also induce ANCA formation. ${ }^{3-5}$

The arthropathy associated with GD is characterized by soft tissue swelling and periosteal bone changes of the fingers, toes, and lower extremities, causing clubbing (Figures 1 and 2).

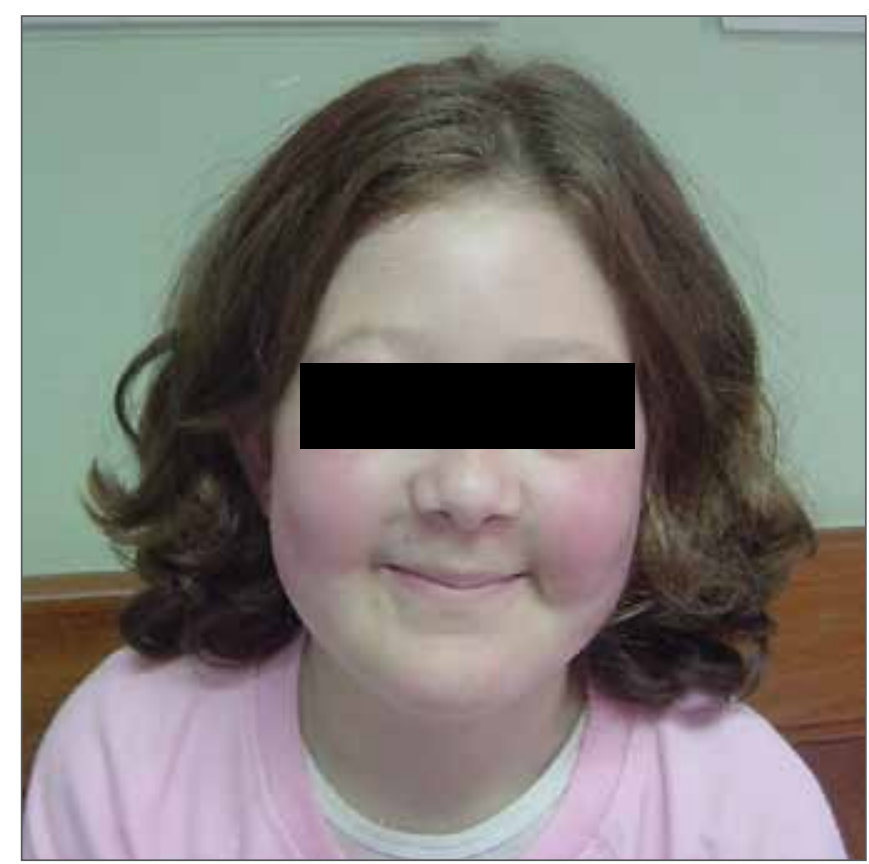

Figure 1

Child's face. Exophthalmos. Malar flush.

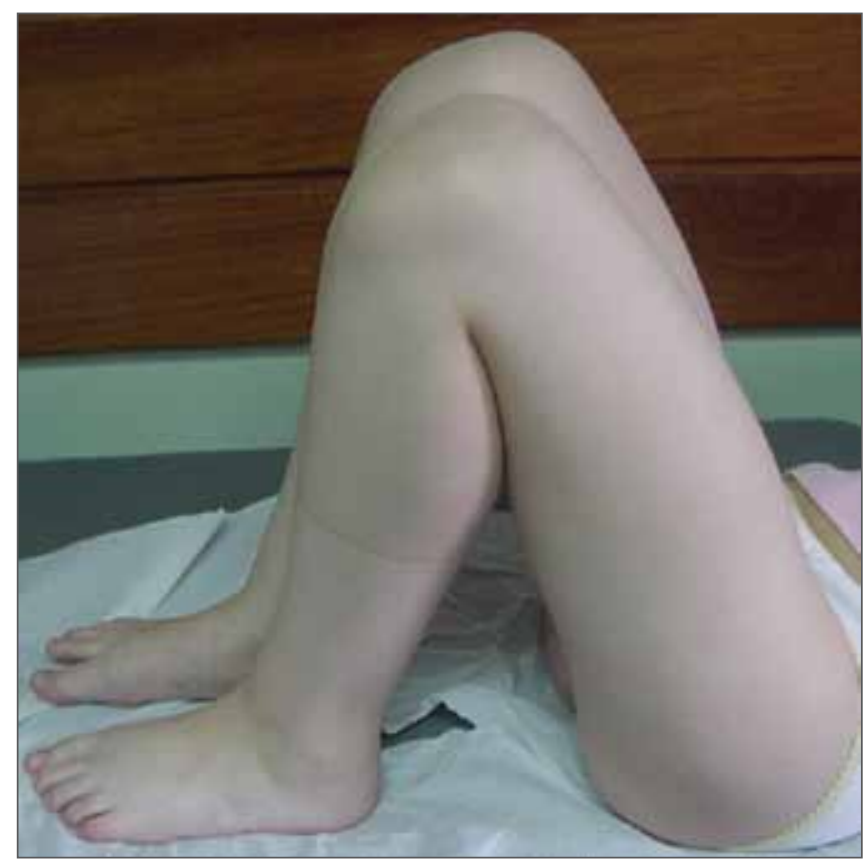

Figure 2

Lower limbs. Limitation of knee flexion (sequelae).

It usually occurs up to 30 years after the initial diagnosis of GD, and is more likely to be seen in patients treated with radioiodine ablation. ${ }^{6}$ Our patient had neither clinical nor radiological signs of thyroid acropachy. 
We consider JIA the most probable hypothesis and chorioretinitis could have been its first presentation, supported by the second episode of uveitis. Also supporting this idea is the fact that autoimmune thyroid disease seems to be associated with other immunologic disorders, including adult-onset RA. They share a common pathophysiology, such as loss of self tolerance, immune tissue destruction caused by several factors, and simultaneous occurrence in the family, suggesting a common genetic background or environmental factors. The single-nucleotide polymorphism (SNP) in the protein tyrosine phosphatase gene N22 (PTPN22) has recently been shown to be associated with an increased risk of developing RA, as well as other autoimmune diseases, such as autoimmune thyroid diseases and SLE, ${ }^{7,8}$ but it was not tested in our patient.

Other SNPs within the IL2RA/CD25 gene previously associated with type 1 diabetes mellitus, GD, and multiple sclerosis have been recently associated with JIA., ${ }^{9,10}$

Bourikas et al. ${ }^{11}$ have reported the concomitance of RA and GD in an adult woman and GD in her sister. Those authors discuss causes of inflammatory arthritis and the case illustrates the genetic predisposition to autoimmunity.

This case illustrates the difficulties encountered in the differential diagnosis of an inflammatory arthritis that develops in a child with GD. 